\section{Tempo de recuperação ao dano muscular induzido por exercícios de força em idosos: uma revisão sistemática}

\author{
Time to muscle recovery from damage after \\ strength exercise in older people: \\ a systematic review
}

\author{
Alexandre Konig Garcia Prado ${ }^{1,2}$ \\ José Claudio Jambassi Filho ${ }^{1,2}$ \\ Marilia Ceccato ${ }^{1,3}$ \\ Luiza Herminia Gallo ${ }^{1,2}$ \\ André Luiz Demantova Gurjão ${ }^{1,3}$ \\ Sebastião Gobbi ${ }^{1}$
}

\section{Resumo}

O processo de recuperação ao dano muscular precisa ser considerado na prescrição adequada de um programa de exercícios de força $(\mathrm{EF})$ para idosos. Inúmeros fatores podem determinar o comportamento desse processo. O objetivo do presente estudo foi realizar uma revisão sistemática sobre o tempo de recuperação ao dano muscular após o EF em idosos. A busca foi realizada de novembro a dezembro de 2011, sem limites de datas na Biological Abstracts, Web of Science, Sports Discus, e PubMed. Uma busca manual também foi realizada nas listas de referências dos estudos encontrados. Oito estudos determinaram o tempo para a completa recuperação ao dano muscular em idosos saudáveis (média de idade $\geq 60$ anos) após a realização de EF. Todos utilizaram métodos indiretos de avaliação do dano muscular, sendo que dentre eles, as avaliações da funcionalidade do sistema neuromuscular ocorreram em maior número. $\mathrm{O}$ EF pode causar danos musculares em idosos que se recuperam entre 24 e 216 horas. Nenhum dos estudos comparou a recuperação ao dano muscular após EF com diferentes ações musculares. Apenas um estudo analisou o efeito da carga repetida sobre o tempo de recuperação ao dano muscular em idosos. As influências de cada variável do EF, do gênero, do treinamento com pesos, do nível de atividade física e do método utilizado para quantificar o dano na recuperação devem ser mais bem estudadas em idosos.

\section{Palavras-chave}

Treinamento de resistência; Manifestações neuromusculares; Envelhecimento.

\begin{abstract}
The recovery process from muscle damage should be taken into consideration in an adequate program of strength exercise (SE) prescription for older adults. Several factors may determine the course of this process. The purpose of this study was to conduct a systematic review regarding the time to recovery from muscle damage after SE in older people. The search was conducted from November to December 2011, without limits of date in Biological Abstracts, Web of Science, Sports Discus, and PubMed. A manual search was also conducted in the reference lists of the selected articles. Eight studies determined the time to complete muscle recovery from damage in healthy elderly (mean age $\geq 60$ years) after SE. All of the studies employed indirect methods of muscle damage assessment, and among those, evaluations of the functionality of the neuromuscular system occurred in greater number. The SE may cause muscle damage in older people that recover between 24 and 216 hours. No study compared recovery from damage after workouts with different muscle actions. Only one study analyzed the repeated bout influence on the time to recovery from muscle damage in older people. Influences of each SE variable, gender, weight training, physical activity status, and the method used to quantify the damage in recovery should be further studied in older people.
\end{abstract}

\section{Keywords}

Resistance training; Neuromuscular manifestations; Aging.
Rev Bras Ativ Fis Saúde p. 156-167 DOI:

http://dx.doi.org/10.12820/rbafs.v.18n2p156

1 Instituto de Biociências, UNESP - Univ Estadual Paulista, Departamento de Educação Física, Laboratório de Atividade Física e Saúde (LAFE), 2 - Bolsista Capes, 3 - Bolsista CNPq. 


\section{INTRODUÇÃO}

A realização de exercícios de força (EF) é um importante estímulo para induzir alterações agudas no sistema neuromuscular ${ }^{1}$. O dano ocorrido no músculo após a realização de $\mathrm{EF}$ é caracterizado por alterações ultraestruturais no sistema musculoesquelético ${ }^{2-4}$, aumento da atividade sérica de enzimas musculares ${ }^{5-6}$, dor muscular tardia (DMT) ${ }^{6-7}$ e decréscimo da função muscular ${ }^{8-9}$.

Diferentes estudos têm indicado que as respostas agudas e a cinética da recuperação ao dano muscular podem ser influenciadas por fatores como gênero ${ }^{10-11} \mathrm{e}$ estado de treinamento ${ }^{8,12}$, bem como pela manipulação das variáveis do EF (Ex.: intensidade ${ }^{13}$, velocidade de execução ${ }^{14}$ e tipo de ação muscular $\left.{ }^{15}\right)$. Adicionalmente, a idade do indivíduo tem sido apontada como um possível fator determinante no grau do dano muscular bem como no processo de recuperação, sendo que indivíduos idosos podem levar maior tempo para se recuperar ${ }^{16}$.

Smith e Norris ${ }^{17}$, ao analisarem estudos com diferentes faixas etárias, indicaram que dependendo do estímulo ofertado, a recuperação pode ocorrer em períodos menores à 24 horas ou até maiores do que 28 dias. Diferentes tempos para recuperação ao dano muscular após EF têm sido demonstrados em idosos. Hakkinen ${ }^{18}$, por exemplo, ao analisar a contração voluntária isométrica máxima (CVIM) - medida utilizada na avaliação da funcionalidade do sistema muscular após EF - apontou que são necessárias 48 horas para a recuperação desse parâmetro após a realização de cinco séries de 10 repetições excêntricas $(\mathrm{EXC})$ e concêntricas $(\mathrm{CON})$ dos flexores dos joelhos e quadris. Por outro lado, Dedrick e Clarkson ${ }^{19}$ encontraram que a CVIM ainda não havia se recuperado após 120 horas da realização de 24 ações EXC dos flexores de cotovelo. Da mesma forma, Ferri et al. ${ }^{1}$ concluíram que os valores de creatina quinase $(\mathrm{CK})$, outro marcador indireto de dano muscular, retornaram aos valores basais após 96 horas da realização de 10 séries de 10 repetições de ações EXC e CON dos flexores plantares. Em contrapartida, Clarkson e Dedrick ${ }^{5}$ indicaram 120 horas para a recuperação desse mesmo marcador após a realização de 24 ações EXC dos flexores de cotovelo. Diferenças relacionadas aos protocolos de exercícios empregados em cada estudo podem ter sido determinantes na diferença entre as horas de recuperação encontradas.

Sabe-se que o processo de recuperação ao dano muscular tem papel fundamental como componente do ciclo de adaptação, uma vez que a completa recuperação pós-treinamento é essencial para melhorar e otimizar o desempenho durante o exercício ${ }^{20}$, sendo um importante fenômeno na prescrição de programas de EF para indivíduos idosos. Fell e Williams ${ }^{16}$ completam que a capacidade do tecido se reparar e se preparar para um estresse irá determinar a frequência e a intensidade do EF. No entanto, parece haver uma variedade de fatores que podem determinar o comportamento do processo de recuperação ao dano muscular e explicar, por consequência, parte das contradições observadas entre os diferentes estudos.

Assim, o objetivo do presente estudo foi realizar uma revisão sistemática na tentativa de verificar o período necessário para a recuperação ao dano muscular, induzido por meio da realização de EF em adultos idosos, bem como discutir os potenciais fatores que podem influenciar neste processo.

\section{MÉTODOS}

A busca eletrônica foi realizada no período de novembro a dezembro de 2011, sem limites de datas, nas seguintes bases de dados: Biological Abstracts, Web of Science, 
Sport Discus, e PubMed. Para a prospecção dos estudos, as palavras-chaves foram utilizadas individualmente e de forma combinada por meio dos operadores booleanos (and e or) da seguinte maneira: recovery AND muscle damage OR muscle injury AND strength exercise OR resistance exercise OR weight exercise AND elderly OR aging OR older adults. Uma busca adicional foi realizada utilizando os termos "repeated-bout exercise" e "repeated-bout effect" individualmente e de forma combinada aos termos "elderly", "aging" e "older adults". Buscas manuais de possíveis estudos relevantes também foram realizadas nas listas de referências de todas as publicações inclusas.

Foram selecionados somente os artigos que atenderam aos seguintes critérios de inclusão: a) amostra composta por adultos idosos saudáveis (média de idade $\geq 60$ anos); b) utilização de, no mínimo, uma medida de avaliação relacionada à recuperação ao dano muscular. Foram excluídos os estudos com: a) animais; b) idosos em reabilitação; c) idosos com limitações articulares ou musculares; e d) ausência de comparações estatísticas (pré e pós-exercício) entre os marcadores de dano muscular.

Levando-se em consideração que as principais evidências do surgimento de dano muscular têm ocorrido após a realização de exercícios que envolvam ações $\mathrm{EXC}^{15,21} \mathrm{e}$, que muitos estudiosos têm utilizado essas ações como forma de induzir o dano muscular ${ }^{5-6,9,19}$, determinou-se que todos os estudos incluídos na presente revisão deveriam incluir ações $\mathrm{EXC}$ em seus protocolos de exercício. Uma vez que ações puramente EXC raramente ocorrem de forma isolada nos movimentos hu$\operatorname{manos}^{22}$, e que a prescrição de EF normalmente não envolve a realização de ações exclusivamente EXC, estudos que realizaram ações CON e EXC também foram incluídos na presente revisão.

Estudos com ações exclusivamente isométricas não foram incluídos para análise na presente revisão, pois este tipo de ação está relacionada a uma maior demanda metabólica, sendo que raramente resultam em dano muscular in vivoo ${ }^{23}$. Por outro lado, medidas de força isométrica têm sido amplamente empregadas para se determinar a função do músculo após a realização de exercícios com ações musculares excêntricas ${ }^{1,9,19}$. Nesse sentido, na presente revisão, ações isométricas foram consideradas apenas quando utilizadas como medidas indiretas de dano muscular.

Os marcadores indiretos de dano muscular avaliados na presente revisão foram: concentração de mioglobina $(\mathrm{Mb})$; atividade sérica de CK e lactato desidrogenase (LDH); pico de torque isocinético (PTISOC); pico de torque isométrico (PTISOM); CVIM; uma repetição excêntrica máxima (1RMEXC); uma repetição concêntrica máxima (1RMCON); taxa de desenvolvimento de força (TDF); máximo valor da integral da atividade eletromiográfica (iEMGMAX); taxa de desenvolvimento da atividade eletromiográfica (TDEMG); DMT; amplitude de movimento (ADM); ângulo articular do cotovelo flexionado (AACFLEX); ângulo articular do cotovelo relaxado (AACRELAX); ângulo ótimo para a produção de força (AOPF); circunferência do braço (CIRC); espessura muscular (ESPMUS) e; intensidade do sinal de ultrassom (IUS). Para estas variáveis, a recuperação foi considerada quando os valores do momento pós-exercício retornaram significativa e consistentemente aos do pré-exercício (linha de base).

Após a realização de uma sessão de exercícios excêntricos intensos parece ocorrer uma redução acentuada dos sintomas de dano muscular na sessão subsequente ${ }^{24-25}$, sendo esse fenômeno conhecido como o efeito da carga repetida ("repeated-bout exercise" ou "repeated bout effect"). Do ponto de vista crônico, a realização de um treinamento com pesos que envolva a prática de ações excêntricas também parece 
causar uma redução da magnitude do dano muscular ${ }^{7-8}$. Dada a importância desses fatores na compreensão do fenômeno da recuperação ao dano muscular, estudos que analisaram o efeito da carga repetida ou do treinamento com pesos na recuperação ao dano muscular de idosos também foram incluídos na presente revisão.

\section{RESULTADOS}

O resumo da busca eletrônica é apresentado na figura 1. Em síntese, 1487 artigos (90 na Biological abstracts, um na Web of Science, 1117 na Sports Discus, 234 na PubMed e 45 por busca manual) foram localizados. Posteriormente à análise baseada nos critérios de inclusão e exclusão, oito estudos foram incluídos nesta revisão.

Artigos localizados inicialmente $=1487$

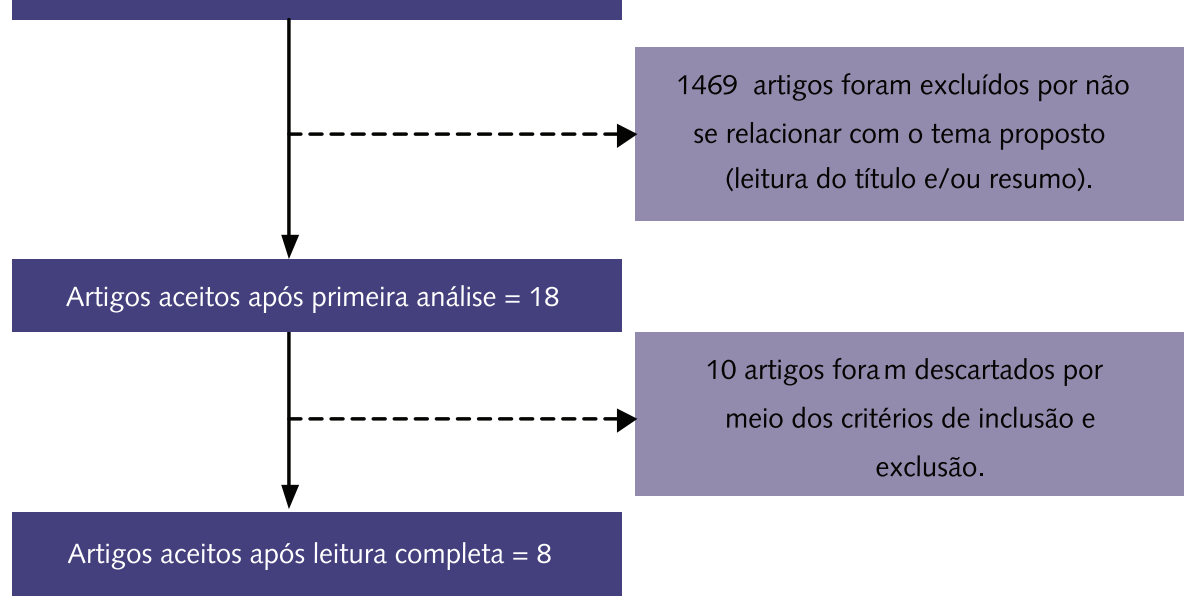

Figura 1 - Resumo da pesquisa na literatura.

A Tabela 1 apresenta um resumo da amostra e protocolo de exercício dos oito estudos encontrados na presente revisão.

A Tabela 2 apresenta um resumo dos parâmetros avaliados, momentos das avaliações e resultados dos estudos encontrados.

As amostras dos estudos foram compostas de seis a 11 participantes. A amplitude das médias de idade dos participantes variou entre 64 e 72 anos. Em nenhum estudo foi empregada amostra composta por ambos os gêneros, dificultando a comparação da recuperação ao dano muscular entre mulheres e homens idosos.

Todos os estudos encontrados avaliaram o dano muscular por meio de marcadores indiretos, sendo que avaliações da funcionalidade do sistema neuromuscular foram as mais utilizadas, sendo encontradas em seis estudos $1,5,8-9,18-19$. Os resultados do estudo de Ferri et al. ${ }^{1}$ demonstraram que o PTISOC, dependendo da velocidade em que é avaliado, pode não se alterar $\left(60^{\circ} \mathrm{s}^{-1}\right)$ ou demorar 24 horas para se recuperar $\left(120^{\circ} \mathrm{s}^{-1}\right.$ e $\left.-60^{\circ} \mathrm{s}^{-1}\right)$. Outras expressões da força dinâmica, como a força concêntrica máxima $(1 \mathrm{RMCON})$ e a força excêntrica máxima (1RME$\mathrm{XC})$, levaram respectivamente 216 e 168 horas para se recuperar em indivíduos sem experiência em treinamento com pesos. Avaliações da força isométrica máxima, determinadas pela CVIM e pelo PTISOM podem não sofrer alteração significativa ou levar mais de 120 horas para se recuperar. A TDF não se alterou significativamente no único estudo que avaliou essa variável. A iEMGMAX não retornou consistentemente para os valores pré-exercício após 48 horas, enquanto a TDEMG não se alterou significativamente. 
Tabela 1 - Resumo dos estudos incluídos na revisão com relação aos sujeitos e protocolo de exercício.

\begin{tabular}{|c|c|c|}
\hline Autor (s) / (ano) & Sujeitos & Protocolo de exercício \\
\hline Ferri et al. (2006) ${ }^{1}$ & 9 o (72 \pm 4 anos) fisicamente ativos & $\begin{array}{l}\text { Exercício: ações EXC/CON isotônicas dos flexores plantares } \\
\text { ADM: } 50^{\circ} \\
\text { Séries: } 10 \\
\text { Repetições: } 10 / \text { série } \\
\text { Intervalo de recuperação: } 90 \mathrm{~s} \\
\text { Intensidade: } 70 \% \text { de } 1 \mathrm{RM} \\
\text { Velocidade: } 17^{\circ} \mathrm{s}^{-1}\end{array}$ \\
\hline $\begin{array}{l}\text { Hakkinen } \\
(1995)^{18}\end{array}$ & $8+(69 \pm 3$ anos $)$ fisicamente ativas & $\begin{array}{l}\text { Exercício: ações EXC/CON isotônicas dos flexores dos joelhos e quadril } \\
\text { ADM: não informado } \\
\text { Séries: } 5 \\
\text { Repetições:10/série } \\
\text { Intervalo de recuperação: } 180 \mathrm{~s} \\
\text { Intensidade: } 70 \% \text { de } 1 \text { RM } \\
\text { Velocidade: não informada }\end{array}$ \\
\hline $\begin{array}{l}\text { Valkeinen et al. } \\
(2006)^{7}\end{array}$ & $\begin{array}{l}11 \text { q (64 } \pm 3 \text { anos) pré e pós } 21 \\
\text { semanas de treinamento com pesos }\end{array}$ & $\begin{array}{l}\text { Exercício ações EXC/CON isotônicas dos extensores da perna } \\
\text { ADM: } 110^{\circ} \\
\text { Séries: } 5 \\
\text { Repetições: } 10 / \text { série } \\
\text { Intervalo de recuperação: } 120 \mathrm{~s} \\
\text { Intensidade: } 100 \% \text { de } 10 \mathrm{RM} \\
\text { Velocidade: não informada }\end{array}$ \\
\hline $\begin{array}{l}\text { Chapman et al. } \\
(2008)^{9}\end{array}$ & $10 \widehat{\partial}(64 \pm 1$ anos $)$ & $\begin{array}{l}\text { Exercício: ações EXC isocinéticas dos flexores de cotovelo } \\
\text { ADM: } 120^{\circ} \\
\text { Séries: } 5 \\
\text { Repetições: } 6 / \text { série } \\
\text { Intervalo de recuperação: } 90 \mathrm{~s} \\
\text { Intensidade: } 100 \% \text { de } 6 \mathrm{RM} \\
\text { Velocidade: } 210^{\circ} \mathrm{s}^{-1}\end{array}$ \\
\hline $\begin{array}{l}\text { Clarkson e } \\
\text { Dedrick }(1988)^{5}\end{array}$ & $\begin{array}{l}10 \text { \& ( } 67 \pm 5 \text { anos }) \text { fisicamente ativas } \\
\text { em duas sessões de EF separadas por } \\
7 \text { dias }\end{array}$ & $\begin{array}{l}\text { Exercício: ações EXC isotônicas dos flexores de cotovelo } \\
\text { ADM: } 105^{\circ} \\
\text { Séries: } 24 \\
\text { Repetições: } 1 / \text { série } \\
\text { Intervalo de recuperação: } 12 \mathrm{~s} \\
\text { Intensidade: } 115 \% \text { da CVIM } \\
\text { Velocidade: } 35^{\circ} \mathrm{s}^{-1}\end{array}$ \\
\hline $\begin{array}{l}\text { Dedrick e } \\
\text { Clarkson }(1990)^{19}\end{array}$ & 10 q (67 \pm 5 anos) fisicamente ativas & $\begin{array}{l}\text { Exercício: ações EXC isotônicas dos flexores de cotovelo } \\
\text { ADM: } 105^{\circ} \\
\text { Séries: } 24 \\
\text { Repetições: } 1 / \text { série } \\
\text { Intervalo de recuperação: } 12 \mathrm{~s} \\
\text { Intensidade: } 115 \% \text { da CVIM } \\
\text { Velocidade: } 35^{\circ} \mathrm{s}^{-1}\end{array}$ \\
\hline $\begin{array}{l}\text { Krishnan et al. } \\
(2003)^{6}\end{array}$ & $80^{\lambda}(66 \pm 2$ anos $)$ sedentários & $\begin{array}{l}\text { Exercício: ações EXC isotônicas para extensores da perna e supino reto } \\
\text { ADM: não informada } \\
\text { Séries: } 10 \\
\text { Repetições: } 10 / \text { série } \\
\text { Intervalo de recuperação: não informado } \\
\text { Intensidade: } 100 \% \text { de } 3 \text { RM } \\
\text { Velocidade: } 3 \text { s/repetição }\end{array}$ \\
\hline $\begin{array}{l}\text { Ploutz-Snyder et } \\
\text { al. }(2001)^{8}\end{array}$ & $\begin{array}{l}6 \text { + }(66 \pm 5 \text { anos }) \text { sedentárias pré e } \\
\text { pós12 semanas de treinamento com } \\
\text { pesos }\end{array}$ & $\begin{array}{l}\text { Exercício: ações EXC isotônicas para extensores do joelho } \\
\text { ADM: não informada } \\
\text { Séries: } 10 \\
\text { Repetições: } 10 / \text { séries } \\
\text { Intervalo de recuperação: } 120 \mathrm{~s} \\
\text { Intensidade: } 75 \% \text { de } 1 \mathrm{RM} \\
\text { Velocidade: não informada }\end{array}$ \\
\hline
\end{tabular}

1RMCON=1 repetição concêntrica máxima; $1 \mathrm{RMEXC}=1$ repetição excêntrica máxima; $A D M=$ Amplitude de movimento; $C O N=$ Concêntrico; $C V I M=$ Contração voluntária isométrica

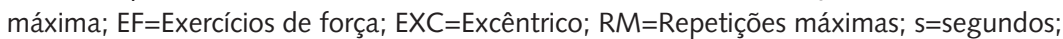
$\hat{\jmath}=$ homens; + =mulheres. 
Tabela 2 - Resumo dos estudos incluídos na revisão com relação aos parâmetros avaliados, momentos das avaliações e resultados.

\begin{tabular}{|c|c|c|c|}
\hline \multirow{2}{*}{$\begin{array}{l}\text { Autor (s) } \\
\text { (ano) }\end{array}$} & \multirow{2}{*}{$\begin{array}{l}\text { Parâmetros } \\
\text { avaliados }\end{array}$} & \multirow{2}{*}{$\begin{array}{l}\text { Momentos das avaliações pós- } \\
\text { exercício }\end{array}$} & Resultados \\
\hline & & & Retornaram consistentemente para os valores pré-exercício após: \\
\hline \multirow{5}{*}{$\begin{array}{l}\text { Ferri et al. } \\
(2006)^{1}\end{array}$} & PTISOC & $5 \mathrm{~min}, 24$ e $48 \mathrm{~h}$ & $\begin{array}{l}24 \mathrm{~h} \text { a velocidade de } 120^{\circ} \mathrm{s}^{-1} \text { e }-60^{\circ} \mathrm{s}^{-1} \text {. Não se alterou } \\
\text { significativamente a } 60^{\circ} \mathrm{s}^{-1}\end{array}$ \\
\hline & PTISOM & $5 \mathrm{~min}, 24$ e $48 \mathrm{~h}$ & Não se alterou significativamente \\
\hline & CK & $1,48,96$ e $144 \mathrm{~h}$ & $96 \mathrm{~h}$ \\
\hline & LDH & $1,48,96$ e $144 \mathrm{~h}$ & $96 \mathrm{~h}$ \\
\hline & $\mathrm{Mb}$ & $1,48,96$ e $144 \mathrm{~h}$ & $48 \mathrm{~h}$ \\
\hline \multirow{4}{*}{$\begin{array}{l}\text { Hakkinen } \\
(1995)^{18}\end{array}$} & CVIM & Imediatamente após, 1, 2, 24 e 48 h & $48 \mathrm{~h}$ \\
\hline & TDF & Imediatamente após, 1, 2, 24 e 48 h & Não se alterou significativamente \\
\hline & iEMGMÁX & Imediatamente após, 1, 2, 24 e 48 h & Não recuperada após 48 h \\
\hline & TDEMG & Imediatamente após, 1, 2, 24 e 48 h & Não se alterou significativamente \\
\hline \multirow{2}{*}{$\begin{array}{l}\text { Valkeinen et al. } \\
(2006)^{7}\end{array}$} & DMT PRÉ-TP & $24,48,72,96,120$ e $154 \mathrm{~h}$ & $72 \mathrm{~h}$ \\
\hline & DMT PÓS-TP & $24,48,72,96,120$ e $154 \mathrm{~h}$ & $72 \mathrm{~h}$ \\
\hline \multirow{9}{*}{$\begin{array}{l}\text { Chapman et al. } \\
(2008)^{9}\end{array}$} & CVIM & $1,24,48,72$ e $96 \mathrm{~h}$ & $96 \mathrm{~h}$ \\
\hline & AOPF & $1,24,48,72$ e $96 h$ & $48 \mathrm{~h}$ \\
\hline & ADM & $1,24,48,72$ e $96 h$ & $96 \mathrm{~h}$ \\
\hline & DMT & $1,24,48,72$ e $96 \mathrm{~h}$ & $96 \mathrm{~h}$ \\
\hline & CIRC & $1,24,48,72$ e $96 \mathrm{~h}$ & Não recuperado após 96 h \\
\hline & IUS & $1,24,48,72$ e $96 \mathrm{~h}$ & Não se alterou significativamente \\
\hline & ESPMUS & $1,24,48,72$ e $96 \mathrm{~h}$ & Não recuperado após 96 h \\
\hline & CK & 24 e 96 h & Não se alterou significativamente \\
\hline & LDH & 24 e $96 \mathrm{~h}$ & Não se alterou significativamente \\
\hline \multirow{4}{*}{$\begin{array}{l}\text { Clarkson } \\
\text { e Dedrick } \\
(1988)^{5}\end{array}$} & AACRELAX & $\begin{array}{l}\text { Imediatamente após, } 24,48,72,96 \\
\text { e } 120 \mathrm{~h}\end{array}$ & 72 h para $1^{\mathrm{a}}$ sessão; 48 h para $2^{\mathrm{a}}$ sessão \\
\hline & AACFLEX & $\begin{array}{l}\text { Imediatamente após, 24, 48, 72, } 96 \\
\text { e } 120 \mathrm{~h}\end{array}$ & 48 h para $1^{\mathrm{a}}$ sessão; 24 h para $2^{\mathrm{a}}$ sessão \\
\hline & CK & $24,48,72,96$ e $120 \mathrm{~h}$ & $\begin{array}{l}\text { Não recuperado } 120 \mathrm{~h} \text { após } 1^{\mathrm{a}} \text { sessão; não se alterou } \\
\text { significativamente após } 2^{\mathrm{a}} \text { sessão }\end{array}$ \\
\hline & DMT & $24,48,72,96$ e $120 \mathrm{~h}$ & $96 \mathrm{~h}$ para $1^{\mathrm{a}}$ sessão; não se alterou significativamente após $2^{\mathrm{a}}$ sessão \\
\hline $\begin{array}{l}\text { Dedrick e } \\
\text { Clarkson } \\
(1990)^{19}\end{array}$ & CVIM & $\begin{array}{l}\text { Imediatamente após, 24, 48, 72, } 96 \\
\text { e } 120 \mathrm{~h}\end{array}$ & Não recuperado após $120 \mathrm{~h}$ \\
\hline \multirow{2}{*}{$\begin{array}{l}\text { Krishnan et al. } \\
(2003)^{6}\end{array}$} & DMT & 24 e $36 \mathrm{~h}$ & Não recuperado após 36 h \\
\hline & CK & $48 \mathrm{~h}$ & Não recuperado após 48 h \\
\hline \multirow{4}{*}{$\begin{array}{l}\text { Ploutz-Snyder } \\
\text { et al. }(2001)^{8}\end{array}$} & 1RMCON PRÉ-TP & $24,72,96,168,216$ e $264 \mathrm{~h}$ & $216 \mathrm{~h}$ \\
\hline & 1RMCON PÓS-TP & $24,72,96,168,216$ e 264 h & $72 \mathrm{~h}$ \\
\hline & 1RMEXC PRÉ-TP & $24,72,96,168,216$ e $264 \mathrm{~h}$ & $168 \mathrm{~h}$ \\
\hline & 1RMEXC PÓS-TP & $24,72,96,168,216$ e $264 \mathrm{~h}$ & $72 \mathrm{~h}$ \\
\hline
\end{tabular}

$1 \mathrm{RMCON}=1$ repetição concêntrica máxima; $1 \mathrm{RMEXC}=1$ repetição excêntrica máxima; $A A C F L E X=A ̂ n-$ gulo da articulação do cotovelo flexionado; AACRELAX=Ângulo da articulação do cotovelo relaxado;

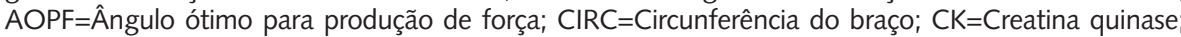
CVIM=Contração voluntária isométrica máxima; DMT=Dor muscular tardia; ESPMUS=Espessura muscular; $h=$ horas; iEMGMÁX=Máximo valor da integral da atividade eletromiográfica; min=minutos; IUS= Intensidade do sinal de ultra-som; $\mathrm{LDH}=$ Lactato desidrogenase; $M b=$ Mioglobina; $P O ́ S-T P=$ Após período de treinamento com pesos; PRE-TP= Antes período de treinamento com pesos; $\mathrm{PTISOC}=\mathrm{Pico}$ de torque isocinético; PTISOM= Pico de torque isométrico; RM=Repetições máximas; s=segundos; TDEM$\mathrm{G}=$ Taxa de desenvolvimento da atividade eletromiográfica; TDF=Taxa de desenvolvimento de força.

Outra forma para se avaliar a funcionalidade do sistema muscular é por meio da ADM ou ângulo articular. Os estudos encontrados demonstraram que os ân- 
gulos articulares e ADM sofreram alteração significativa após EF e se recuperam entre 24 e 96 horas. $\mathrm{O}$ AOPF se alterou em aproximadamente $25^{\circ}$ e retornou aos valores iniciais após 48 horas.

Medidas de circunferência e espessura muscular foram utilizadas em apenas um estudo, e retornaram aos valores pré-exercício após 96 horas. A intensidade do sinal de ultrassom tem sido utilizada também como marcador indireto de dano, mas não se alterou em nenhum momento após o EF. A avaliação da DMT, o marcador indireto de dano muscular mais utilizado na literatura, segundo Warren et al. ${ }^{26}$, foi encontrada em quatro dos oito estudos, com sua recuperação ocorrendo entre 72 e 96 horas após o EF.

Outras medidas indiretas de dano muscular encontradas foram as de atividade sérica de enzimas musculares. Estas foram utilizadas em quatro estudos 1, 5-6, ${ }^{9}$, os quais demonstraram que os níveis de CK não se alteraram em alguns casos ou indicaram a presença de dano muscular até 120 horas pós-exercício. O nível de LDH, outro indicador de condição da membrana das células do músculo esquelético, pode não se alterar ou demorar até 96 horas para retornar aos valores pré-exercício. A concentração de $\mathrm{Mb}$, analisada em apenas um estudo, retornou aos níveis pré-exercício após 48 horas.

Dos estudos encontrados aqui, dois ${ }^{7-8}$ procuraram verificar o efeito do treinamento na recuperação muscular. No estudo de Ploutz-Snyder et al. ${ }^{8}$, o tempo total de treinamento foi de 12 semanas e acontecia duas vezes por semana, consistindo na realização de 8-12 repetições de extensão-flexão bilateral de joelhos até a fadiga. $\mathrm{Na}$ média, os participantes treinaram a $65 \%$ de $1 \mathrm{RM}$ concêntrica. Já no estudo de Valkeinen et al. ${ }^{7}$, o treinamento teve duração de 21 semanas e ocorria duas vezes por semana. Cada sessão incluía dois exercícios para os músculos extensores do joelho e quatro a cinco exercícios para os principais grupamentos musculares do corpo. A intensidade do exercício aumentou progressivamente de $50 \%$, inicialmente, até $80 \%$ de $1 \mathrm{RM}$ ao final do treinamento. No estudo de Valkeinen et al. ${ }^{7}$, embora o tempo de recuperação para DMT tenha sido o mesmo antes e após o período de treinamento, os autores apontaram uma diminuição considerável nos níveis de dor após a realização do treinamento. Já no estudo de Ploutz-Snyder et al. ${ }^{8}$ fica evidente um menor tempo necessário para recuperar tanto a força CON (CON 1RM) quanto a EXC dinâmica máxima (EXC 1RM) após período de treinamento.

Quatro dos oito estudos encontrados tinham a amostra composta por indivíduos fisicamente ativos, sendo que cada estudo determinou o nível de atividade física por meio de um método diferente. Nenhum estudo incluiu indivíduos já treinados com pesos em sua amostra, sendo que três estudos ${ }^{7-9}$ adotaram como critério de exclusão a participação em programas de EF estruturados. Um único estudo tinha indivíduos sedentários como sujeitos.

\section{DISCUSSÃO}

A análise dos oito artigos encontrados demonstrou que a realização de EF pode causar danos musculares em idosos que podem exigir até 216 horas para completa recuperação do sistema muscular. No entanto, dada a grande possibilidade de avaliações do dano muscular e os inúmeros fatores que podem influenciar neste fenômeno, os resultados destes oito estudos não permitem uma conclusão inequívoca a respeito da recuperação ao dano muscular após EF em idosos.

Uma ampla variedade de medidas para avaliação do dano muscular em idosos após EF foi encontrada, sendo que as avaliações da funcionalidade do sistema 
muscular foram as mais empregadas. Segundo Warren e Armstrong ${ }^{26}$, em estudo que revisou as ferramentas de medição usadas no estudo de dano muscular induzido por contração excêntrica, a utilização de medidas como a CVIM e amplitude de movimento, que indicam a funcionalidade do sistema muscular, proporcionaram os melhores métodos para quantificar o dano muscular. Esse tipo de medida oferece um benefício adicional ao praticante de exercícios, fornecendo a real medida de funcionalidade do sistema, sendo esta mais relacionada ao desempenho durante o exercício.

Medidas como a atividade sérica de enzimas musculares foram utilizadas em $50 \%$ dos estudos encontrados na presente revisão. De uma forma geral, o tempo de recuperação para essas variáveis se demonstrou inconsistente entre os diferentes estudos. Outro estudo que procurou avaliar o dano muscular por meio da atividade sérica de enzimas musculares, como a $\mathrm{CK}$, demonstrou uma alta variabilidade dessa medida ${ }^{27}$. Além disso, Manfredi et al. ${ }^{2}$ observaram não haver relação significativa entre a atividade sérica de CK e a correspondente quantidade de dano muscular indicada pela análise em microscópio eletrônico e de luz, sugerindo que a CK pode ser um fraco indicador de dano muscular induzido pelo exercício.

Tanto para as variáveis utilizadas na avaliação da funcionalidade do sistema muscular quanto da atividade sérica de enzimas musculares, os resultados dos diferentes estudos encontrados aqui parecem não concordarem. Inúmeros fatores podem explicar a grande amplitude de períodos encontrados para a completa recuperação ao dano muscular. Possivelmente, a principal causa pode estar relacionada aos protocolos de exercícios empregados em cada situação.

Diferentes estudos têm indicado que ações musculares EXC resultam em um maior dano muscular do que ações $\mathrm{CON}$ ou isométricas ${ }^{15,21}$. Provavelmente, o maior dano muscular após ações EXC deve-se à combinação de uma maior capacidade de gerar força e menor recrutamento das fibras musculares, gerando um alto estresse mecânico nas estruturas envolvidas ${ }^{28}$. Neste contexto, os estudos que procuram induzir o dano muscular e avaliar o processo de recuperação têm aplicado ações EXC.

$\mathrm{Na}$ presente revisão foi identificado que, dos estudos que avaliaram a recuperação ao dano muscular de idosos após a realização de $\mathrm{EF}$, mais de $60 \%$ utilizaram ações exclusivamente EXC. Krishnan et al. ${ }^{6}$ demonstraram que, em indivíduos idosos sedentários, após a realização de exercícios exclusivamente $\mathrm{EXC}$ isotônicos para membros inferiores, a DMT não havia se recuperado dentro de 36 horas, bem como a CK após 48 horas. Ploutz-Snyder et al. ${ }^{8}$, também após exercícios exclusivamente $\mathrm{EXC}$ isotônicos para membros inferiores, encontraram que a capacidade de gerar força excêntrica dinâmica máxima se recuperou somente após 168 horas. Quando os exercícios envolveram os membros superiores, resultados divergentes foram encontrados. Três estudos ${ }^{5,9,19}$ empregaram exercícios EXC para flexores do cotovelo. Chapman et al. ${ }^{9}$ e Dedrick \& Clarkson ${ }^{19}$ encontraram tempos de recuperação para a CVIM de 96 e 120 horas respectivamente, enquanto a recuperação da DMT ocorreu após 96 horas em ambos os estudos. Com relação à ADM, Clarkson \& Dedrick ${ }^{5}$ encontraram menor tempo de recuperação comparada ao estudo de Chapman et al. ${ }^{9}$. No estudo de Clarkson \& Dedrick ${ }^{5}$, os níveis de CK não haviam retornado aos valores pré-exercício mesmo após 120 horas, enquanto que no estudo de Chapman et al. ${ }^{9}$, não foi identificada alteração significativa para essa mesma variável em avaliações após 24 e 96 horas.

Mesmo limitando-se apenas à realização de ações EXC, as divergências encontradas no tempo de recuperação desses marcadores podem estar relacionadas às 
diferenças entre outras variáveis dos protocolos empregados nestes cinco estudos, como por exemplo, a velocidade de execução. Chapman et al. ${ }^{9}$ utilizaram alta velocidade de contração $\left(210^{\circ} \mathrm{s}^{-1}\right)$ enquanto Dedrick e Clarkson ${ }^{19}$ e Clarkson e Dedrick $^{5}$ utilizaram velocidade de contração baixa $\left(35^{\circ} \mathrm{s}^{-1}\right)$. Diferentes estudos têm demonstrado que a velocidade da contração do exercício pode afetar diretamente o tempo de recuperação dos marcadores indiretos de dano muscular ${ }^{14,29}$. Nenhum dos estudos encontrados na presente revisão procurou verificar a influência da velocidade de execução na recuperação ao dano muscular após EF. Ressalta-se também que as influências da manipulação de outras variáveis agudas do EF no processo de recuperação ao dano muscular não têm sido estudada na literatura, principalmente na população idosa.

Outro fator que pode ter gerado divergências entre os resultados dos estudos supracitados é o fato destes serem realizados com diferentes gêneros. Flores et al. ${ }^{10}$, após quatro dias do final de uma sessão de EF, demonstraram uma dinâmica diferente no processo de recuperação ao dano muscular entre gêneros. Estudos têm especulado que a diferença nos níveis de dano muscular entre os gêneros podem estar relacionadas às concentrações de estrógeno. Sugere-se que esse hormônio, encontrado em maiores níveis nas mulheres, pode exercer um papel protetor, minimizando o dano muscular ${ }^{30-31}$.

Deve-se chamar a atenção para a aplicação de ações EXC de forma isolada com o intuito de se induzir o dano muscular, uma vez que essa pode não ser a melhor forma de se representar uma sessão de exercícios comumente empregada. Ações musculares EXC raramente ocorrem de forma isolada nos movimentos naturais do ser humano ${ }^{22}$, que por sua vez, são caracterizados por sequencias de ações EXC seguidas por ações CON, conhecidas como o ciclo de alongamento e encurtamento ${ }^{32}$. Assim, estudos que empreguem protocolos de exercícios que envolvam ações EXC e CON poderão proporcionar maior validade ecológica, devendo ser realizados para melhor compreensão da recuperação ao dano muscular de indivíduos idosos após a realização de EF comumente empregados.

$\mathrm{Na}$ presente revisão, apenas três estudos que realizaram exercícios com ações EXC e CON foram encontrados ${ }^{1,7,18}$. Com relação à recuperação da DMT, essa foi observada após 72 horas e, para a CK e LDH foram necessários 96 horas. A TDF e TDEMG não se alteraram significativamente após o exercício e a iEMGMÁX ainda não havia se recuperado após 48 horas. O PTISOC avaliado nas velocidades de $120^{\circ} \mathrm{s}^{-1} \mathrm{e}-60^{\circ} \mathrm{s}^{-1}$ se recuperou após 24 horas, sendo que na velocidade de $60^{\circ} \mathrm{s}^{-1}$ não se alterou significativamente. Quarenta e oito horas foi o tempo necessário para recuperação dos níveis de $\mathrm{Mb}^{1}$. Com relação à capacidade de realizar força isométrica máxima, não foi encontrada alteração significativa no estudo de Ferri et al. ${ }^{1}$, enquanto que essa mesma variável levou 48 horas para se recuperar no estudo de Hakkinen ${ }^{18}$. Tal contradição pode estar associada à diferença no grau ou recuperação do dano muscular entre gêneros, uma vez que os estudos foram realizados em gêneros diferentes.

Com relação aos estudos que envolveram a participação de indivíduos idosos em um programa de treinamento com $\operatorname{pesos}^{7-8}$, foi demonstrado que esse pode influenciar no processo de recuperação ao dano muscular, diminuindo o tempo de recuperação e causando menor DMT. Os autores sugerem que o treinamento pode causar uma proteção contra subsequentes esforços não costumeiramente realizados, incluindo aqueles de alta intensidade e maior número de repetições do que normalmente realizado durante o treinamento. Uma limitação desses estudos foi a análise de um baixo número de variáveis relacionados à recuperação ao dano muscular. 
Outro importante fator relacionado à recuperação ao dano muscular, é que a realização de exercícios com músculos já danificados pode não aumentar o dano ou afetar negativamente o processo de recuperação muscular ${ }^{33-35}$. Assim, após a realização de uma sessão de exercícios excêntricos intensos parece ocorrer uma redução da magnitude do dano muscular, conhecido como o efeito da carga repetida. Investigações sobre os mecanismos do efeito da carga repetida sugerem que esse processo ocorre por meio de uma mudança no padrão de recrutamento da fibra muscular, pelo fortalecimento do tecido conjuntivo, ou através de adaptações celulares que reduzem a tensão do sarcômero dentro do músculo ${ }^{35}$.

Dos estudos encontrados na presente revisão, apenas um procurou verificar o efeito da carga repetida no tempo de recuperação de indivíduos idosos ${ }^{5}$. Os autores demonstraram que após sete dias da realização de uma sessão de exercícios EXC de alta intensidade, a realização de uma nova sessão com o mesmo protocolo de exercícios não aumentou o dano causado pela sessão anterior. $\mathrm{O}$ tempo de recuperação foi reduzido após a segunda sessão para as medidas de DMT, CK, ângulos articulares flexionados e relaxados. Outro estudo ${ }^{36}$ também observou o efeito da carga repetida em indivíduos idosos, no entanto, os autores não avaliaram se o tempo de recuperação ao dano muscular foi afetado após a segunda sessão de exercícios, fazendo com que o estudo fosse excluído da presente revisão.

Muito pouco ou nada se sabe a respeito da influência do nível de atividade física na recuperação ao dano muscular após EF. Nenhum dos estudos encontrados procurou comparar a recuperação de idosos sedentários com idosos fisicamente ativos.

Dessa forma, fica clara uma ampla variedade de fatores que podem influenciar no processo de recuperação ao dano induzido pelo EF em pessoas idosas. A literatura ainda é contraditória com relação ao exato papel que cada fator exerce, sendo que novos estudos procurando verificar uma possível influência da manipulação de cada variável do EF, do gênero e do nível de atividade física devem ser realizados com indivíduos idosos.

Por meio da análise dos oito artigos que atenderam a todos os critérios de inclusão, podemos concluir que, após a realização de uma sessão de EF, podem ser necessários desde algumas horas até vários dias para a completa recuperação do sistema neuromuscular de indivíduos idosos. Embora pareça evidente que exercícios EXC de alta intensidade exijam maior tempo para recuperação muscular ao dano, outras variáveis relacionadas à prescrição do EF devem ser manipuladas e comparadas dentro de protocolos que representem um cenário real e comum de treinamento para indivíduos idosos. Avaliações da funcionalidade do sistema muscular como medidas indiretas de dano muscular têm sido amplamente empregadas e devem ser preferidas em detrimento daquelas relacionadas a níveis séricos de enzimas musculares. Mulheres e homens podem necessitar de períodos diferentes para se recuperar do dano muscular, no entanto, possíveis influências do gênero na recuperação ao dano muscular de idosos após EF ainda não foram estudadas. Conquanto, de uma forma geral, pareça claro na literatura o efeito positivo do treinamento na recuperação ao dano, estudos com idosos ainda são escassos e necessitam analisar um maior número de variáveis relacionadas à recuperação ao dano muscular. Após a realização de uma sessão de exercícios excêntricos intensos parece ocorrer uma redução do tempo de recuperação ao dano muscular em sessões de exercícios posteriores.

\section{Agradecimentos}

Laboratório de Atividade Física e Envelhecimento (LAFE), Conselho de Aperfeiçoamento de Pessoal de Nível Superior (CAPES), Conselho Nacional de Desenvolvimento Científico e Tecnológico (CNPq). 


\section{Contribuição dos Autores}

Todos os autores do manuscrito são membros do LAFE, atuando como coordenadores ou professores, sendo alunos de pós-graduação que desenvolvem pesquisas para Dissertações de Mestrado ou Tese de Doutorado. Todos colaboraram integralmente com a elaboração do manuscrito, tanto na fase de busca dos artigos quanto na redação do mesmo.

\section{REFERÊNCIAS}

1. Ferri A, Narici M, Grassi B, Pousson M. Neuromuscular recovery after a strength training session in elderly people. Eur J Appl Physiol 2006;97:272-9.

2. Manfredi TG, Fielding RA, O'Reilly KP et al. Plasma creatine kinase activity and exerciseinduced muscle damage in older men. Med Sci Sports Exerc 1991;23:1028-34.

3. Roth SM, Martel GF, Ivey FM, et al. Ultrastructural muscle damage in young vs. older men after high-volume, heavy-resistance strength training. J Appl Physiol 1999;86:1833-40.

4. Roth SM, Martel GF, Ivey FM, et al. High-volume, heavy-resistance strength training and muscle damage in young and older women. J Appl Physiol 2000;88:1112-8.

5. Clarkson PM, Dedrick ME. Exercise-induced muscle damage, repair, and adaptation in old and young subjects. J Gerontol 1988;43:M91-6.

6. Krishnan RK, Evans WJ, Kirwan JP. Impaired substrate oxidation in healthy elderly men after eccentric exercise. J Appl Physiol 2003;94:716-23.

7. Valkeinen H, Hakkinen A, Hannonen P, Hakkinen K, Alen M. Acute heavy-resistance exercise-induced pain and neuromuscular fatigue in elderly women with fibromyalgia and in healthy controls: effects of strength training. Arthritis Rheum 2006;54:1334-9.

8. Ploutz-Snyder LL, Giamis EL, Formikell M, Rosenbaum AE. Resistance training reduces susceptibility to eccentric exercise-induced muscle dysfunction in older women. J Gerontol A Biol Sci Med Sci 2001;56:B384-90.

9. Chapman DW, Newton M, Mcguigan MR, Nosaka K. Comparison between old and young men for responses to fast velocity maximal lengthening contractions of the elbow flexors. Eur J Appl Physiol 2008;104:531-539.

10. Flores DF, Gentil P, Brown LE, et al. Dissociated time course of recovery between genders after resistance exercise.J Strength Cond Res 2011;25:3039-44.

11. Sewright KA, Hubal MJ, Kearns A, Holbrook MT, Clarkson PM. Sex differences in response to maximal eccentric exercise. Med Sci Sports Exerc 2008;40:242-51.

12. Fernandez-Gonzalo R, Bresciani G, Souza-Teixeira F, et al. Effects of a 4-week eccentric training program on the repeated bout effect in young active women. J Sports Sci Med 2011;10:692-699.

13. Chen TC, Nosaka K, Sacco P. Intensity of eccentric exercise, shift of optimum angle, and the magnitude of repeated-bout effect. J Appl Physiol 2007;102:992-999.

14. Chapman D, Newton M, Sacco P, Nosaka K. Greater muscle damage induced by fast versus slow velocity eccentric exercise. Int J Sports Med 2006;27:591-8.

15. Golden CL, Dudley GA. Strength after bouts of eccentric or concentric actions. Med Sci Sport Exer 1992; 24:926-933.

16. Fell J, Williams AD. The effect of aging on skeletal-muscle recovery from exercise: Possible implications for aging athletes. J Aging Phys Activ 2008;16:97-115.

17. Smith DJ, Norris SR. M. Kellmann. Enhancing recovery: Preventing underperformance in athletes. Champaign, IL: Human Kinetics, 2002: 81-101

18. Hakkinen K. Neuromuscular fatigue and recovery in women at different ages during heavy resistance loading. Electromyogr Clin Neurophysiol 1995;35:403-13.

19. Dedrick ME, Clarkson PM. The effects of eccentric exercise on motor performance in young and older women. Eur J Appl Physiol Occup Physiol 1990;60:183-6.

20. Bishop PA, Jones E, Woods AK. Recovery from training: a brief review. J Strength Cond Res 2008;22:1015-24.

21. Newham DJ, Mcphail G, Mills KR, Edwards RH. Ultrastructural changes after concentric and eccentric contractions of human muscle. Journal of the Neurological Science 1983;61:109-122. 
22. Byrne C, Twist C, Eston R. Neuromuscular function after exercise-induced muscle damage: theoretical and applied implications. Sports Med 2004;34:49-69.

23. Close GL, Kayani A, Vasilaki A, Mcardle A. Skeletal muscle damage with exercise and aging. Sports Med 2005;35:413-427.

24. Skurvydas A, Brazaitis M, Kamandulis S. Repeated bout effect is not correlated with intraindividual variability during muscle-damaging exercise. J Strength Cond Res 2011;25:1004-1009.

25. Venckunas T, Skurvydas A, Brazaitis M, et al. Human alpha-actinin-3 genotype association with exercise-induced muscle damage and the repeated-bout effect. Appl Physiol Nutr Metab 2012;37:1038-1046.

26. Warren GL, Lowe DA, Armstrong RB. Measurement tools used in the study of eccentric contraction-induced injury. Sports Med 1999;27:43-59.

27. Arnett MG, Hyslop R, Dennehy CA, Schneider CM. Age related variations of serum creatine kinase and creatine kinase mioglobin response in females. Can J Appl Physiol 2000;25:419-429.

28. Enoka RM. Eccentric contractions require unique activation strategies by the nervous system. J Appl Physiol 1996;81:2339-46.

29. Ellwanger RB, Brentano MA, Kruel LFM. Efeito da utilização de diferentes velocidades do treino de força em marcadores indiretos de lesão muscular. Rev Bras Educ Fís Esp 2007; 21:259-270.

30. Tiidus PM. Can estrogens diminish exercise induced muscle damage? Can J Appl Physiol 1995;20:26-38.

31. Clarkson PM, Sayers SP. Etiology of exercise-induced muscle damage. Can J Appl Physiol 1999;24:234-248.

32. Komi PV. Stretch-shortening cycle: a powerful model to study normal and fatigued muscle. J Biomech 2000;33:1197-206.

33. Clarkson PM, Nosaka K, Braun B. Muscle function after exercise-induced muscle damage and rapid adaptation. Med Sci Sport Exer 1992;24:512-20.

34. Nosaka K, Clarkson PM. Muscle damage following repeated bouts of high force eccentric exercise. Med Sci Sport Exer 1995;27:1263-9.

35. Mchugh MP, Connolly DA, Eston RG, Gleim GW. Exercise-induced muscle damage and potential mechanisms for the repeated bout effect. Sports Med 1999;27:157-70.

36. Lavender AP, Nosaka K. Responses of old men to repeated bouts of eccentric exercise of the elbow flexors in comparison with young men. Eur J Appl Physiol 2006;97 619-626.

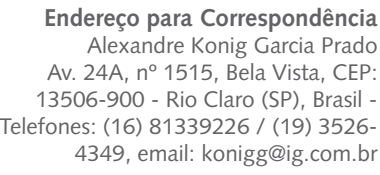

Recebido 08/06/2012

Revisado 14/10/2012

Aprovado 07/11/2012 